\title{
Seletracetam (UCB 44212)
}

\author{
Barbara Bennett,* Alain Matagne, ${ }^{\dagger}$ Philippe Michel, ${ }^{\ddagger}$ Michèle Leonard, ${ }^{\S}$ Miranda Cornet, ${ }^{\S}$ \\ Marie-Anne Meeus, ${ }^{\text {II }}$ and Nathalie Toublanc ${ }^{\text {TI }}$ \\ *CNS Clinical Development, UCB Atlanta, Smyrna, Georgia 30080; ${ }^{\dagger}$ Preclinical CNS, ${ }^{\ddagger}$ Chemistry, ${ }^{\S}$ Non-clinical Development, \\ and ${ }^{\mathrm{I}}$ Clinical Pharmacology, UCB Braine-l'Alleud, Belgium
}

\begin{abstract}
Summary: Better pharmacotherapies for epilepsy are needed for patients who are refractory to or have tolerability difficulties with current treatments. Seletracetam, a new drug in epilepsy development, is a pyrrolidone derivative structurally related to levetiracetam (trade name Keppra). It was discovered because of its high binding affinity to the synaptic vesicle $2 \mathrm{~A}$ (SV2A) protein, which is now known to be the binding site for this family of compounds. Seletracetam shows very potent seizure suppression in models of acquired or genetic epilepsy, as well as high CNS tolerability in various animal models. Pharmacokinetic studies in animals suggest that seletracetam is rapidly and highly absorbed, with linear and time-independent pharmacokinetics. Seletracetam appears neither to inhibit nor to induce the major human drug metabolizing enzymes, and it
\end{abstract}

demonstrates low plasma protein binding $(<10 \%)$, which suggests a low potential for drug-drug interactions. Initial studies in humans demonstrated first-order monocompartmental kinetics with a half-life of $8 \mathrm{~h}$ and an oral bioavailability of $>90 \%$. Studies in healthy volunteers showed that the treatment emergent adverse events were of mild to moderate severity, were mostly of CNS origin and were resolved within $24 \mathrm{~h}$. Altogether, these results suggest that seletracetam represents a promising new antiepileptic drug candidate, one that demonstrates a potent, broad spectrum of seizure protection and a high CNS tolerability in animal models, with initial clinical findings suggestive of straightforward pharmacokinetics and good tolerability. Key Words: Seletracetam, epilepsy, SV2A, pharmacokinetics, kindled, anticonvulsant

\section{INTRODUCTION}

\section{Epilepsy and the need for new antiepileptic drugs}

Epilepsy is a chronic, often lifelong, neurological disorder that requires continuous pharmacotherapy to maintain seizure control. The age-adjusted incidence of epilepsy in developed countries is approximately 50 per 100,000 persons per year, ${ }^{1}$ with a lifetime chance of getting epilepsy of $3-5 \% .^{2}$ Epilepsy has a tremendous impact not only on the individuals afflicted with this disease, but also on their families, as well as on society in general, because of the resultant personal and financial burdens. Epilepsy is characterized by recurrent involuntary seizures, caused by disturbances in the normal electrical activity of the brain, that affect awareness, movement, or sensations. ${ }^{3}$ These seizures may occur in just one area of the brain (partial seizures) or may affect neuronal networks throughout the brain (generalized seizures).

Address correspondence and reprint requests to: Barbara A. Bennett, Ph.D., UCB, Inc., Clinical Development, Neurology/Psychiatry Therapeutic Area, 1950 Lake Park Drive, Smyrna, GA 30080. E-mail: Barbara.bennett@ucb-group.com.
Pharmacotherapy is the primary treatment for epileptic patients; however, despite the introduction of secondgeneration anticonvulsants, refractory epilepsy is still a significant clinical problem. Outcome studies examining either traditional or newer anticonvulsants show that fewer than $50 \%$ of patients with epilepsy become seizure-free after beginning an initial antiepileptic medication. ${ }^{4}$ Approximately $30 \%$ of patients (adults and children) with partial onset seizures cannot be adequately controlled with existing antiepileptic drugs (AEDs), because of either lack of efficacy or toxicity. ${ }^{5}$ Most refractory patients have partial epilepsy; the generalized epilepsies tend to respond better to current AED therapy. ${ }^{6}$ Furthermore, these refractory patients are at risk for cognitive and psychiatric disorders, suicide, accidental injuries, and sudden death. There continues to be a need for new antiepileptic drugs, both to improve upon the established drugs and also to treat those refractory to current pharmacotherapies.

\section{Current drugs for the treatment of epilepsy}

Classical (or first-generation) anticonvulsants, such as phenobarbital, phenytoin, carbamazepine, and valproate, 




FIG. 1. Structural formula for seletracetam.

are used as standard therapy in partial epilepsy. Clinical problems associated with these drugs include nonlinear pharmacokinetics, narrow therapeutic index, significant drug interactions, and adverse central nervous system effects. $^{7}$ The entrance to the market of vigabatrin, felbamate, lamotrigine, oxcarbazepine, topiramate, zonisamide, gabapentin, levetiracetam, tiagabine, and pregabalin increased the therapeutic options and improved treatment. However, most of these new drugs have a limited tolerability profile. Serious toxicity problems have been identified, including fatal idiosyncratic hepatic failure and aplastic anemia with felbamate, fatal allergic rashes with lamotrigine, and serious visual field defects with vigabatrin. New AEDs with improved risk-benefit ratios are therefore needed for refractory epilepsy patients, ${ }^{8}$ both as adjunctive treatment and in monotherapy.

\section{SELETRACETAM}

\section{Preclinical beginnings}

Seletracetam is a small-molecule AED (FIG. 1), nonionized in water, and with solubility properties not expected to lead to any solubility- or dissolution-related absorption problems after oral administration.

\section{Pharmacological background}

Seletracetam is a structural analog of the antiepileptic drug levetiracetam, and its pharmacological activity appears to relate principally to an interaction with a novel binding site, synaptic vesicle protein $2 \mathrm{~A}$ (SV2A). ${ }^{9,10}$ Seletracetam binds selectively, stereospecifically, and with high affinity (10-fold greater affinity than levetiracetam) to SV2A, which is thought to be involved with synaptic vesicle exocytosis and neurotransmitter release. Both classic AEDs, such as carbamazepine, phenytoin, valproate, phenobarbital, and clonazepam, and new AEDs, such as gabapentin, tiagabine, vigabatrin, felbamate, and zonisamide, are devoid of significant affinity for SV2A. (S)-stereoisomer analogs of levetiracetam show a rank order of affinity for $\left[{ }^{3} \mathrm{H}\right]$ levetiracetam binding that correlates with their seizure protection, ${ }^{10,11}$ suggesting a functional correlation between binding and anticonvulsant activity. Seletracetam did not induce a significant displacement ( $\leq 10 \mu \mathrm{mol} / \mathrm{L}$ ) of radioligands specific for the other known binding sites within the CNS (e.g., receptors, uptake systems, and ion channel proteins). ${ }^{9}$ Thus, it appears that SV2A represents a novel molecular target that seems to have an important role in the pharmacological activity of seletracetam.

The effect of seletracetam on several voltage-dependent ionic and receptor-gated currents has been characterized. Seletracetam, like levetiracetam, does not appear to have any effect on voltage-dependent $\mathrm{Na}^{+}$channels. ${ }^{12}$ Likewise, seletracetam $(1-100 \mu \mathrm{mol} / \mathrm{L})$ had no effect on voltage-gated $\mathrm{K}^{+}$currents (both the A-type and the delayed rectifier) recorded in mouse hippocampal neurons in culture. ${ }^{13}$ Seletracetam, like levetiracetam, appears to inhibit high-voltage-activated (HVA) $\mathrm{Ca}^{2+}$ currents, but does not appear to modulate the low-voltage-activated (T-type) $\mathrm{Ca}^{2+}$ currents. ${ }^{14}$ Levetiracetam has been shown to inhibit the N-type channel ${ }^{15}$ and possibly the P-type channel to a much smaller extent. ${ }^{16}$

Seletracetam was found to be devoid of any direct effect on GABA- and strychnine-sensitive glycine-elicited currents, similarly to levetiracetam. ${ }^{17}$ In contradistinction, seletracetam revealed a selectivity toward the glycine receptors (levetiracetam affects both). At pharmacologically relevant concentrations, seletracetam did not modify NMDA-, kainate-, or AMPA-induced currents. These data suggest that the pharmacological properties of seletracetam may derive principally from the potent and selective interaction of seletracetam with the SV2A protein, as well as from the moderate effects of the drug on calcium channels and strychnine-sensitive glycine receptors. The interface between the CNS binding site, SV2A, and these cellular effects is not known; correspondingly, whether any alleged interaction would exist, direct or indirect, is also unknown.

\section{Activity in animal models of seizures and epilepsy}

Seletracetam, like levetiracetam, shows no anticonvulsant activity in the two classical screening tests for AEDs: the maximal electroshock test (MES; tonic convulsions in the hind limbs) and the pentylenetetrazol test (PTZ; generalized clonic convulsions), both acute seizure models. ${ }^{18}$ In distinct contrast, seletracetam shows potent seizure suppression in models of acquired or genetic epilepsy (TABLE 1). ${ }^{18}$ Seletracetam displayed potent protection against secondary generalized motor seizures in fully corneally kindled mice $\left(\mathrm{ED}_{50}\right.$ of $0.31 \mathrm{mg} /$ $\mathrm{kg}$ ) and hippocampally kindled rats (minimal active dose, MAD, of $0.23 \mathrm{mg} / \mathrm{kg}$ ). ${ }^{18}$

The genetically sound-susceptible mouse model is an experimental genetic model of epilepsy, mimicking generalized reflex convulsive epilepsy in humans. Seletracetam provided dose-dependent protection in the genetically sound-susceptible mouse against clonic convulsions induced by an acoustic stimulus with an $\mathrm{ED}_{50}$ of $0.17 \mathrm{mg} / \mathrm{kg}$ (TABLE 1$){ }^{18}$ 
TABLE 1. Antiseizure Effect of Seletracetam in in vivo Animal Models of Seizure and Epilepsy

\begin{tabular}{lll}
\hline \multicolumn{1}{c}{ Model (Object of study) } & Species & \multicolumn{1}{c}{ Dose } \\
\hline Corneal kindling (Generalized motor seizures) & Mouse & $\mathrm{ED}_{50}=0.31 \mathrm{mg} / \mathrm{kg} \mathrm{IP}$ \\
Audiogenic seizures (Clonic convulsions) & Mouse & $\mathrm{ED}_{50}=0.17 \mathrm{mg} / \mathrm{kg} \mathrm{IP}$ \\
GAERS (Suppression of spontaneous SWDs) & Rat & $\mathrm{ED}_{50}=0.15 \mathrm{mg} / \mathrm{kg} \mathrm{IP}$ \\
Hippocampal kindling (Generalized motor seizures) & Rat & MAD $=0.23 \mathrm{mg} / \mathrm{kg}$ PO
\end{tabular}

GAERS $=$ genetic absence epilepsy rats from Strasbourg, MAD = minimal active dose, SWDs $=$ spike-and-wave discharges.

Genetic absence epilepsy rats from Strasbourg (GAERS) represent a strain of Wistar rats, originally selected by inbreeding, that are characterized by the spontaneous occurrence of EEG spike-and-wave discharges (SWDs) concomitant to behavioral arrest. ${ }^{19}$

Based on both neurophysiological and pharmacological characterization of these rats, they appear to fully meet the requirements for an experimental model of nonconvulsive absence epilepsy in humans. Treatment of GAERS rats with seletracetam markedly suppressed their spontaneous SWDs, resulting in an $\mathrm{ED}_{50}$ of 0.15 $\mathrm{mg} / \mathrm{kg}$ i.p. ${ }^{18}$ The results obtained from these two animal models representing primary generalized epilepsies demonstrate a potent and complete seizure suppression by seletracetam.

\section{CNS tolerability in animals}

The rotarod test of Dunham and Miya ${ }^{20}$ is a classical test commonly used for determination and quantification of the minimal neurological deficit. In the rotarod test, seletracetam exhibits an even higher protective index than levetiracetam (the protective index, or the ratio of $\mathrm{TD}_{50}$ for rotarod toxicity to $\mathrm{ED}_{50}$ for seizure protection, is a measure of the separation between anticonvulsant potency and potency to induce motor impairment). ${ }^{21}$ Previous studies revealed a high separation between doses induced significant rotarod impairment and seizure protection in both corneally kindled mice and GAERS rats when levetiracetam was compared with classical and newer AEDs. ${ }^{22}$ Seletracetam impaired rotarod performance in corneally kindled mice $\left(\mathrm{TD}_{50}=325 \mathrm{mg} / \mathrm{kg}\right)$ and GAERS rats $\left(\mathrm{TD}_{50}=449 \mathrm{mg} / \mathrm{kg}\right)$. Compared with the protective $\mathrm{ED}_{50}$ values obtained in the same animals, this resulted in an unusually high CNS tolerability margin of 1048 and 3075, respectively. ${ }^{18}$

\section{Pharmacokinetic profile in animals}

Seletracetam is rapidly and highly absorbed in animals, with linear and time-independent pharmacokinetics. ${ }^{23}$ The exposure to the drug is similar in both sexes in all animal species tested. Plasma protein binding is low, and seletracetam is rapidly distributed to tissues. The major metabolic pathway of seletracetam, which has been observed in all tested species, consists of hydrolysis of the acetamide group to form the carboxylic acid metabolite ucb-101596-1, which is pharmacologically inac- tive. The parent compound, along with its major metabolite, is excreted mainly in urine. In vitro, seletracetam neither inhibits nor induces the major human drug metabolizing CYP isoenzymes and does not inhibit human epoxide hydrolase. ${ }^{24,25}$

\section{Toxicological information}

Seletracetam has a low acute oral toxicity in rat and dog, and presents no significant CNS, cardiopulmonary, or respiratory tract effects, as demonstrated in the safety pharmacology studies. ${ }^{26,27}$ Repeated dosing of seletracetam in rat and dog showed that high doses of 2000 $(\mathrm{mg} / \mathrm{kg}) /$ day in rat and $\geq 600(\mathrm{mg} / \mathrm{kg}) /$ day in dog were not well tolerated. Drug-related effects were observed from dose levels of $20(\mathrm{mg} / \mathrm{kg}) /$ day in rat and $50(\mathrm{mg} /$ $\mathrm{kg}$ )/day in dog. Results of genotoxicity tests give no evidence for seletracetam being genotoxic at any of the doses and concentrations tested. The reproductive toxicology studies performed thus far have shown that seletracetam is neither embryotoxic nor teratogenic. ${ }^{28}$

\section{Pharmacokinetics in humans}

The pharmacokinetics of seletracetam was studied in healthy male subjects in a single rising-dose study, with doses ranging from $2-600 \mathrm{mg}$ (FIG. 2). ${ }^{29}$ Seletracetam was rapidly absorbed, as demonstrated by a maximal plasma concentration $\left(C_{\max }\right)$ that was reached within $1 \mathrm{~h}$ after dosing in the majority of fasted subjects. Coadministration with a high-fat meal resulted in a delayed time to $C_{\max }$ (median time delayed from 0.5 to $4 \mathrm{~h}$ after the dose) and a $39 \%$ reduction in $C_{\max }$, but had no effect on the overall extent of absorption. The disposition of seletracetam was characterized by linear pharmacokinetics over the 300-fold dose range studied.

The volume of distribution is approximately $0.6 \mathrm{~L} / \mathrm{kg}$, which is close to that of total body water. Seletracetam plasma terminal half-life is approximately $8 \mathrm{~h}$ in young healthy male subjects; it does not vary with dose. The total apparent clearance is approximately $67 \mathrm{~mL} / \mathrm{min}$, or approximately $0.8(\mathrm{~mL} / \mathrm{min}) / \mathrm{kg}$; this is much lower than the hepatic blood flow $(1500 \mathrm{~mL} / \mathrm{min})$, indicating a low extraction ratio by the liver. Based on available in vitro data, there is a low potential for interaction of seletracetam on other drugs or of other drugs on seletracetam. Subjects in this study ${ }^{29}$ reported adverse events typical of CNS drugs, including dizziness, somnolence, nausea, 


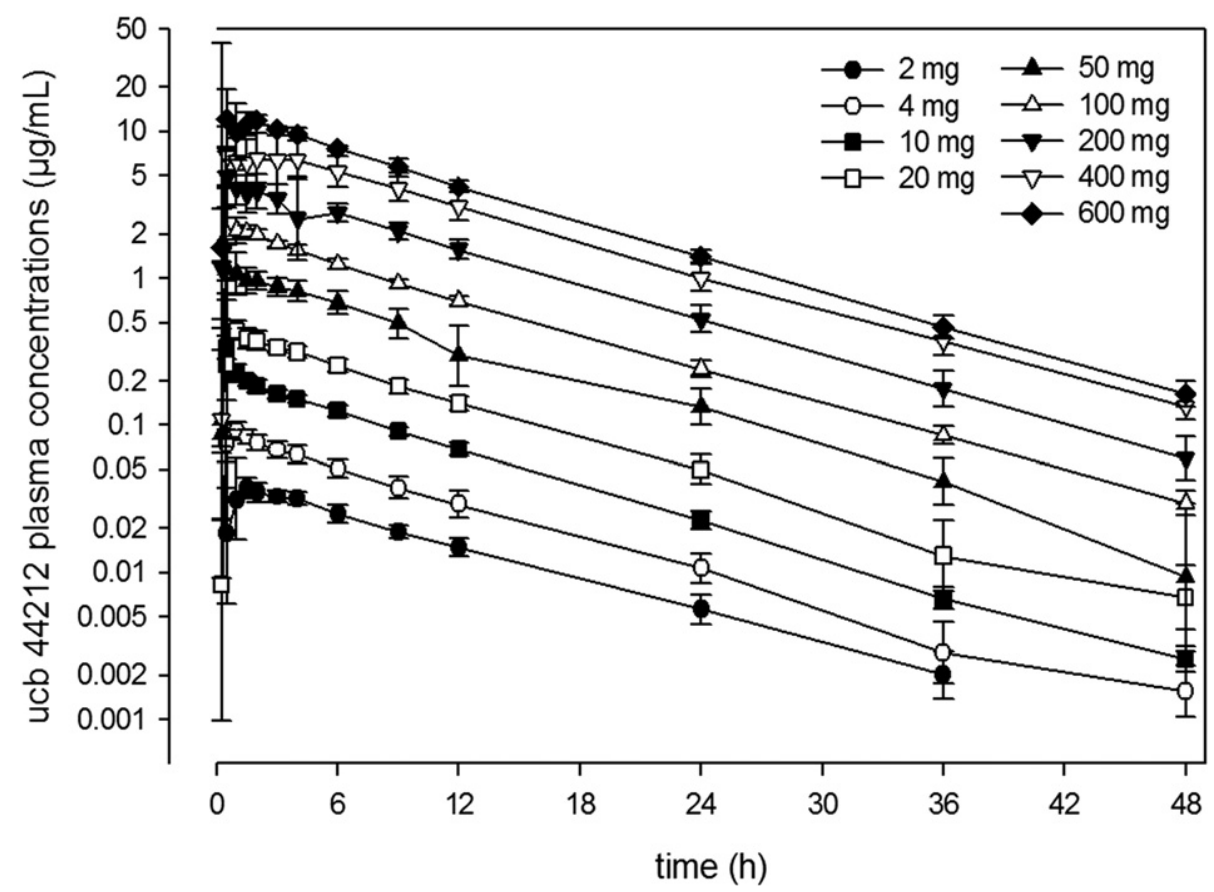

FIG. 2. Mean plasma concentration-time profiles of seletracetam following single oral doses in normal healthy volunteers. Error bars: \pm SD. Results are presented in semi-logarithmic scale.

and headache. The incidence of dizziness tended to increase $\geq 50 \mathrm{mg}$; however, nausea, somnolence, and headache did not seem to be dose-related. All the adverse events reported were of mild or moderate intensity and the doses tested appeared to be well tolerated.

A repeated-dose study was performed in 36 healthy male volunteers. ${ }^{30}$ Subjects received either 20,40 , or 200 $\mathrm{mg}$ bid for 2 weeks. Seletracetam was shown to have predictable pharmacokinetics. Dose proportionality was confirmed for elimination and half-life. The metabolite concentrations were approximately 10 -fold lower than those of the parent compound. After repeated dosing, no unexpected accumulation of either parent compound or metabolite was observed and no autoinduction of seletracetam clearance occurred, confirming time-independent pharmacokinetics. The subject-reported adverse events were similar to those observed in the single risingdose study, with CNS adverse events (AEs) reported most frequently - the majority of which were of mild to moderate intensity. In general, these AEs occurred within $1 \mathrm{~h}$ of initial dosing and decreased in incidence with subsequent dosing.

A study was conducted to investigate the excretion balance and metabolism of orally administered [14C]seletracetam $(100 \mathrm{mg})$ in six healthy male volunteers. ${ }^{31}$ Absorption of seletracetam administered as an oral solution is rapid and nearly complete (recovery of $92 \%$ of radioactive tracer in urine). The urine metabolic profiling indicated that, besides the parent compound, one major metabolite was identified (ucb-101596-1, the acidic metabolite) and two additional minor metabolites.
At $168 \mathrm{~h}, 3.21 \%$ of the dose was recovered in the feces and $91.9 \%$ of the dose was recovered in urine, reflecting a high absorption of the drug. In the urine, the unchanged compound $(25 \%)$ and the major metabolite measured $(53 \%)$ represented $78 \%$ of the dose.

In plasma, radioactivity was measurable until $72 \mathrm{~h}$ after the dose for all subjects. The major radioactive component was the parent drug, representing generally more than $90 \%$ of the circulating radioactivity up to $24 \mathrm{~h}$ after administration. The remaining radioactivity (up to $10 \%$ ) was associated with ucb-101596-1, the acidic metabolite.

The blood cell/plasma ratio (0.5-0.7), computed up to $24 \mathrm{~h}$, indicates some distribution with rapid and reversible equilibrium and no retention of [14C]-seletracetam in blood cells. Protein binding of radioactivity accounted for less than $10 \%$ at 1,6 , and $24 \mathrm{~h}$ after the dose. Overall, these results confirmed the pharmacokinetic parameters observed from previous studies. The AEs were mild and similar to those observed in the single and multiple rising-dose studies, with CNS adverse events reported most frequently.

\section{AED therapy: efficacy versus tolerability}

Given the apparent lack of difference in efficacy among AEDs, tolerability plays a key role when selecting AED therapy. ${ }^{32}$ However, approximately $20-30 \%$ of patients fail anticonvulsant therapy because of intolerable AEs. ${ }^{33}$ Although complete seizure freedom is generally thought to be the most significant predictor of improved quality of life, ${ }^{32}$ AEs may be the most important 
negative influence on a person's perception of individual current health status. ${ }^{34}$ Indeed, significant AEs associated with established AEDs are estimated to contribute to initial treatment failure in $>40 \%$ of patients. ${ }^{34}$ The preclinical data obtained with seletracetam suggest a superior profile differentiating efficacious versus CNS-impairing doses that portends for a very potent anticonvulsant with a high CNS tolerability.

\section{CONCLUSIONS}

Seletracetam was chosen from a large portfolio of SV2A ligands based not only on its higher affinity for this binding site (10-fold greater affinity for SV2A than levetiracetam), but also on its potency and efficacy as observed in in vivo and in vitro testing. The mechanism whereby binding to SV2A results in anticonvulsant activity is as yet unknown. Like levetiracetam, seletracetam is not active in the traditional MES or PTZ models, but shows a potent ability to suppress seizure activity in animal models of acquired or genetic epilepsy. The linear, time-independent pharmacokinetics of the drug combined with a rapid and almost complete absorption indicate that seletracetam has a very uncomplicated pharmacokinetic profile. The initial human studies also indicate that seletracetam is well tolerated at high doses. Taken together, these results suggest that seletracetam represents a promising new AED candidate that demonstrates a potent and broad spectrum of seizure protection and high CNS tolerability in animal models, with initial clinical findings suggestive of straightforward pharmacokinetics and good tolerability.

\section{REFERENCES}

1. Sander JW. The epidemiology of epilepsy revisited. Curr Opin Neurol 2003;16:165-170.

2. Sander JW, Shorvon SD. Epidemiology of the epilepsies. J Neurol Neurosurg Psychiatry 1996;61:433-443 [Erratum in: J Neurol Neurosurg Psychiatry 1997;62:679].

3. Dreifuss FE. Classification of epileptic seizures. In: Engel J Jr, Pedley TA, editors. Epilepsy: a comprehensive textbook. Vol. 1. Philadelphia: Lippincott Raven Publishers, 1997. p. 517-524.

4. Kwan P, Brodie MJ. Early identification of refractory epilepsy. New Engl J Med 2000;342:314-319.

5. Cockerell OC, Johnson AL, Sander JW, Shorvon SD. Prognosis of epilepsy: a review and further analysis of the first nine years of the British National General Practice Study of Epilepsy, a prospective population-based study. Epilepsia 1997;38:31-46.

6. Mattson RH, Cramer JA, Collins JF; Department of Veterans Affairs Epilepsy Cooperative Studies No. 118 and No. 264 Group. Prognosis for total control of complex partial and secondarily generalized tonic clonic seizures. Neurology 1996;47:68-76.

7. Brodie MJ. Do we need any more new antiepileptic drugs? Epilepsy Res 2001;45:3-6.

8. Brodie MJ, Dichter MA. Antiepileptic drugs. N Engl J Med 1996; 334:168-175 [Erratum in: N Engl J Med 1996;334:479].

9. Fuks B, Bouché F. Affinity of ucb 44212 for levetiracetam binding site (LBS) and its binding profile. UCB report reference code: RRLE03B2402. Brussels: UCB Pharma, 2003.

10. Lynch BA, Lambeng N, Nocka K, et al. The synaptic vesicle protein SV2A is the binding site for the antiepileptic drug levetiracetam. PNAS 2004;101:9861-9866.
11. Noyer M, Gillard M, Matagne A, Hénichart JP, Wülfert E. The novel antiepileptic drug levetiracetam (ucb L059) appears to act via a specific binding site in CNS membranes. Eur J Pharmacol 1995;286:137-146.

12. Zona C, Niespodziany I, Pieri M, Klitgaard H, Margineanu D-G. Seletracetam (ucb 44212), a new pyrrolidone derivative, lacks effect on $\mathrm{Na}^{+}$currents in rat brain neurons in vitro. Epilepsia 2005;46(Suppl 8):116.

13. Matagne A. Electrophysiological examination of ucb 44212 on voltage-gated $\mathrm{K}+$ currents in cultured mouse hippocampal neurons. UCB report reference code: RRLE02G0305. Brussels: UCB Pharma, 2005.

14. Pisani A, Bonsi P, Martella G, Cuomo D, Klitgaard H, Margineanu D. Seletracetam (ucb 44212), a new pyrrolidone derivative, inhibits high-voltage-activated $\mathrm{Ca}^{2+}$ currents and intracellular $\left[\mathrm{Ca}^{2+}\right]$ increase in rat cortical neurons in vitro. Epilepsia 2005;46(Suppl 8): 119 .

15. Lukyanetz EA, Shkryl VM, Kostyuk PG. Selective blockade of N-type calcium channels by levetiracetam. Epilepsia 2002;43: 9-18.

16. Pisani A, Bonsi P, Martella G, et al. Intracellular calcium increase in epileptiform activity: modulation by levetiracetam and lamotrigine. Epilepsia 2004;45:719-728.

17. Rigo JM, Nguyen L, Hans G, et al. Seletracetam (ucb 44212): Effect on inhibitory and excitatory neurotransmission. Epilepsia 2005;46(Suppl 8):110.

18. Matagne A, Margineanu D, Michel P, Kenda B, Klitgaard H. Seletracetam (UCB 44212), a new pyrrolidone derivative, reveals potent activity in in vitro and in vivo models of epilepsy. J Neurol Sci 2005;238(Suppl 1):S133.

19. Marescaux C, Vergnes M, Depaulis A. Genetic absence epilepsy in rats from Strasbourg: a review. J Neural Transm Suppl 1992;35: 37-69.

20. Dunham NW, Miya TS. A note on a simple apparatus for detecting neurological deficit in rats and mice. J Am Pharm Assoc 1957;46: $208-209$

21. Loscher W, Nolting B. The role of technical, biological and pharmacological factors in the laboratory evaluation of anticonvulsant drugs. IV. Protective indices. Epilepsy Res 1991;9:1-10.

22. Klitgaard H, Matagne A, Gobert J, Wulfert E. Evidence for a unique profile of levetiracetam in rodent models of seizures and epilepsy. Eur J Pharmacol 1998;353:191-206.

23. Dubois M, Vaernewijck A. ucb 44212: Pharmacokinetics in the Wistar rat after single oral administration of doses ranging from 3 to $1000 \mathrm{mg} / \mathrm{kg}$. UCB report reference code: RRLE02M1005. Brussels: UCB Pharma, 2003.

24. Ogilvie B. ucb 44212: Evaluation as a potential inhibitor of human microsomal epoxide hydrolase. UCB report reference code: RRLE03G1504. Brussels: UCB Pharma, 2004.

25. Ndikum-Moffor F. In vitro evaluation of ucb 44212 as an inducer of cytochrome P450 expression in cultured human hepatocytes. UCB report reference code: RRLE03F2403. Brussels: UCB Pharma, 2004.

26. Bassett S. ucb 44212: 13-week oral toxicity study in Wistar rats followed by a 4-week recovery period. UCB report reference code: RRLE04B1908. Brussels: UCB Pharma, 2005.

27. Harvey P. ucb 44212: 13-week oral toxicity study in beagle dogs followed by a 4-week recovery period. UCB report reference code: RRLE04B1108. Brussels: UCB Pharma, 2005.

28. Perks DA. ucb 44212: Oral (gavage) embryofoetal development study in the rabbit. UCB report reference code: RRLE04A1211. Brussels: UCB Pharma, 2005.

29. Goldwater DR, Lu Z, Salas V, et al. Seletracetam single rising dose safety, tolerability and pharmacokinetics in healthy subjects. Presented at the European Congress on Epilepsy; July 2-6, 2006; Helsinki.

30. Leese PT, Hulhoven R, Salas V, et al. Seletracetam multiple dose safety, tolerability and pharmacokinetics in healthy subjects. Presented at the European Congress on Epilepsy; July 2-6, 2006; Helsinki.

31. Ramael S, Sargentini-Maier ML, Toublanc N, Dubin GM, Daoust A, Stockis A. Pharmacokinetics and metabolism of ${ }^{14} \mathrm{C}$-seletrac- 
etam in healthy subjects. Presented at the European Congress on Epilepsy; July 2-6, 2006; Helsinki, Finland.

32. Perucca E, Beghi E, Dulac O, Shorvon S, Tomson T. Assessing risk to benefit ratio in antiepileptic drug therapy. Epilepsy Res 2000;41:107-139.

33. Mattson RH, Cramer JA, Collins JF. Early tolerance to antiepilep- tic drug side effects: a controlled trial of 24 patients. In: Frey H-H, Fröscher W, Koella WP, Meinardi H, editors. Tolerance to beneficial and adverse effects of antiepileptic drugs. New York: Raven Press, 1986. p. 149-156.

34. Gilliam F. Optimizing health outcomes in active epilepsy. Neurology 2002;58(Suppl 5):S9-S20. 